\title{
El rol del sexo en el Síndrome Apnea Hipopnea Obstructiva del Sueño (SAHOS), en una cohorte de pacientes del nororiente Colombiano
}

\author{
Mendoza Herrera, T. ${ }^{1,2}$, Parra Gómez. L. A. 2,3,4
}

'Universidad Inqustrial de Santander, " ${ }^{G}$ Grupo de investigación MEOITA, JA Asociación de Sociedades Cientificas de Estudiantes de Medicina de Colombia (ASCEMCOL), ¿Universidad Autónoma de Bucaramanga.

\begin{tabular}{|c|c|c|c|c|c|c|}
\hline \multicolumn{7}{|c|}{ Objetivo: } \\
\hline \multicolumn{7}{|c|}{$\begin{array}{l}\text { Determinar el efecto del sexo en los } \\
\text { rasgos clínicos y de polisomnografía } \\
\text { (PSG) en pacientes con SAHOS del } \\
\text { nororiente Colombiano. }\end{array}$} \\
\hline \multirow{2}{*}{ Sintomas } & \multicolumn{2}{|c|}{ Mujeres } & \multicolumn{3}{|c|}{ Hombres } & \\
\hline & \multicolumn{2}{|c|}{ WH $<15$ ИK $\geq 15$} & \multicolumn{2}{|c|}{ Hस } & & \\
\hline Ronquido & \multicolumn{2}{|c|}{$29(20.7 \%) \quad 32(228 \%) 2$} & $29(20.7 \%)$ & \multicolumn{2}{|c|}{$50(35.7 \%)$} & .036 \\
\hline Fartga durna & \multicolumn{6}{|c|}{$15(17 \%) \quad 24(27.2 \%) \quad 12(13.6 \%) \quad 37(42 \%) \quad 0.492$} \\
\hline Apneas presenciadas & \multicolumn{6}{|c|}{$13(16.85) \quad 21(27.25) 9(11.6 \%) \quad 34(44.15) \quad 0.527$} \\
\hline Cetalea matutina & \multicolumn{3}{|c|}{$20(28.9 \%) 16(23.15) \quad 14(20.2 \%)$} & \multicolumn{2}{|c|}{$19(27.5 \%)$} & 206 \\
\hline Hpersomia & $11(16.9 \%) 2$ & $20(30.7 \%) \quad 13$ & $13(20 \%)$ & \multicolumn{2}{|c|}{$21(32.3 \%)$} & 1962 \\
\hline \multicolumn{7}{|c|}{ 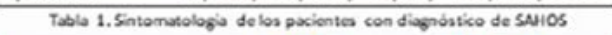 } \\
\hline \multirow{2}{*}{ Caracteristicas } & \multicolumn{2}{|c|}{ Mujeres } & \multicolumn{4}{|c|}{ Hombres } \\
\hline & WH स15 & WH:15 & WH. & ब15 & & 215 \\
\hline Edad & $59,3 \div 11,4$ & $59,4=13,2$ & $3,253,2=1$ & & $53,3=$ & \\
\hline \multirow{5}{*}{$\begin{array}{l}\text { IMC (k9/m2) } \\
\text { Perimetro de cuello } \\
\text { (centimetros) } \\
\text { Perimetro aboominal } \\
\text { (centimetros) } \\
\text { Dumetro mento-esternal } \\
\text { (centimetros) } \\
\text { Escala de EPWORTH }\end{array}$} & $31.2 \pm 6.8$ & $34.3 \pm 7.2$ & $231.8 \pm 5$ & & & \\
\hline & $38.4 \pm 3.3$ & $39 \pm 3.7$ & $43.8 \div 3$ & & $43,4 \pm$ & \\
\hline & $101,3 \pm 10,1$ & $107,6=14$ & $14 \quad 110,5 \pm$ & 13,6 & $107,6=$ & \\
\hline & $14,3 \pm 2,1$ & $15,2 \approx 2,4$ & $4 \quad 14,7 \pm 2$ & & $15,6=$ & \\
\hline & $7,3 \pm 6.3$ & $9.8 \div 67$ & $9.1 \pm 4,4$ & & 12.8. & 7.5 \\
\hline
\end{tabular}

Tabls 2. Carateraticas de los pacientes con diagnóstico de SANOS

\section{Materialesy métodos:}

Estudio observacional analitico tipo corte transversal, con muestreo no probabilístico, en pacientes con somnolencia diuma, remitidos a laboratorios de sueño de la zona metropolitana de Bucaramanga, Colombia. Variables sociodemográficas, clinicas y de PSG conformaron base de datos "INO-SUEÑO13".

\begin{tabular}{|c|c|c|c|c|}
\hline \multirow{2}{*}{$\begin{array}{c}\text { Variables } \\
\text { polisomnograficas }\end{array}$} & \multicolumn{2}{|c|}{ Mujeres } & \multicolumn{2}{|c|}{ Hombres } \\
\hline & IAH $<15$ & $\mathrm{IAH} \geq 15$ & IAH $<15$ & $\angle A H \geq 15$ \\
\hline TST (minutos) & $340,8 \pm 81,2$ & $325 \pm 98,7$ & $354,8 \pm 64,2$ & $331,4 \pm 73,5$ \\
\hline Eficiencia del sueño (\%) & $76,8 \pm 15,4$ & $74,6 \pm 18,4$ & $82 \pm 13,6$ & $77,1 \pm 16,7$ \\
\hline $\begin{array}{l}\text { Saturación media en } \\
\text { vigilia (\%) }\end{array}$ & $92 \pm 3,2$ & $91,7 \pm 3,6$ & $93 \pm 2,1$ & $90,5 \pm 5,7$ \\
\hline T90 (\%) & $20,9 \pm 26,2$ & $17,6 \pm 26,5$ & $23,8 \pm 30,6$ & $11,8 \pm 20,4$ \\
\hline Indice de desaturación & $33,8 \pm 35,2$ & $26,7 \pm 26$ & $25,9 \pm 25,6$ & $34,4 \pm 21,6$ \\
\hline $\begin{array}{l}\text { Indice de despertares en } \\
\text { REM }\end{array}$ & $18 \pm 17,5$ & $30,9 \pm 24,4$ & $30,9 \pm 30,3$ & $24,2 \pm 24,4$ \\
\hline $\begin{array}{l}\text { indice de despertares en } \\
\text { no REM }\end{array}$ & $19,7 \pm 17,1$ & $31,6 \pm 25,9$ & $29,9 \pm 30,2$ & $28,1 \pm 24,9$ \\
\hline
\end{tabular}

Tabla 3. Variables polisomog dificas en pxientes con diagnóntico de SAHOS

\section{Resultados:}

Se analizaron 152 pacientes con diagnóstico de SAHOS (IAH>5); predominantemente hombres $(53,9 \%)$, con edad promedio de 56 años (mayor en mujeres 59,4 años). Se observaron las siguientes comorbilidades; en mujeres: hipotiroidismo $(75 \%)$, falla cardiaca $(59,2 \%)$, dislipidemia $(54,6 \%)$, hipertensión arterial $(54,3 \%)$; en hombres: enfermedad pulmonar obstructiva crónica $(58,8 \%)$, enfermedad coronaria $(55 \%)$, obesidad $(51,6 \%)$. Antecedente de tabaquismo y alcoholismo predominante en hombres (69,9\% y $76,4 \%$ respectivamente), tabaquismo pasivo y exposición a biomasa en mujeres $(59,5 \%$ y $52,6 \%$ respectivamente). Características clínicas en mujeres: parestesia nocturna $(60,4 \%)$ y cefalea matutina $(52,1 \%)$; y nombres: bruxismo $(74 \%)$, somniloquia $(66,6 \%)$, movimiento de piernas $(61,1 \%)$, apneas presenciadas $(55,8 \%)$, fatiga diurna $(55,6 \%)$, ronquido $(52,4 \%)$. Caracteristicas de PSG: tiempo total de sueño promedio $336( \pm 80,3)$ minutos, eficiencia de sueño promedio $77,4 \%( \pm 16,3)$, saturación media/vigilia $91,6 \%( \pm 4,2)$, T90 promedio $19,4 \%( \pm 27)$ e indice de desaturación promedio de $30,4( \pm 26,9:$ mayor $34,4 \pm$ 21,6).

\section{Conclusiones:}

Se observó mayor edad en mujeres con SAHOS, predominio de comorbilidades cardiovasculares, tabaquismo pasivo y exposición a biomasa; con características clinicas atipica de SAHOS, pendiente definir el papel de cefalea matutina e insomnio.

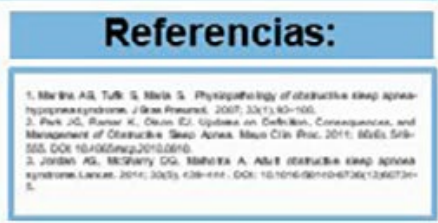

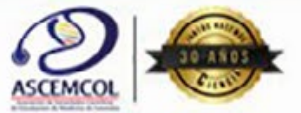

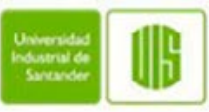

